\title{
Error Bounds and Finite Termination for Constrained Optimization Problems
}

\author{
Wenling Zhao, Daojin Song, and Bingzhuang Liu \\ School of Science, Shandong University of Technology, Zibo, Shandong 255049, China \\ Correspondence should be addressed to Wenling Zhao; zwlsdj@163.com
}

Received 6 February 2014; Accepted 3 April 2014; Published 30 April 2014

Academic Editor: Changzhi Wu

Copyright (C) 2014 Wenling Zhao et al. This is an open access article distributed under the Creative Commons Attribution License, which permits unrestricted use, distribution, and reproduction in any medium, provided the original work is properly cited.

\begin{abstract}
We present a global error bound for the projected gradient of nonconvex constrained optimization problems and a local error bound for the distance from a feasible solution to the optimal solution set of convex constrained optimization problems, by using the merit function involved in the sequential quadratic programming (SQP) method. For the solution sets (stationary points set and $K K T$ points set) of nonconvex constrained optimization problems, we establish the definitions of generalized nondegeneration and generalized weak sharp minima. Based on the above, the necessary and sufficient conditions for a feasible solution of the nonconvex constrained optimization problems to terminate finitely at the two solutions are given, respectively. Accordingly, the results in this paper improve and popularize existing results known in the literature. Further, we utilize the global error bound for the projected gradient with the merit function being computed easily to describe these necessary and sufficient conditions.
\end{abstract}

\section{Introduction}

This paper is concerned with the following constrained optimization problem:

$$
\min \{f(x) \mid x \in S\}
$$

where $f: \mathbf{R}^{n} \rightarrow \mathbf{R}$ is a continuously differentiable function

$$
S=\left\{x \in \mathbf{R}^{n} \mid c_{i}(x) \leq 0, i=1,2, \ldots, m\right\},
$$

and $c_{i}: \mathbf{R}^{n} \rightarrow \mathbf{R}$, for $i=1,2, \ldots, m$, are continuously differentiable convex functions.

It is well known that SQP is an important method for solving the problem $(N P)$. Its essential idea is to approximate the solutions of the problem (NP) by using the optimal solutions of a series of quadratic programming. The solutions here may be referred to as the stationary points, $K K T$ points, or optimal solutions of the problem (NP).

Suppose that $x \in S$ is a current iterate point of the problem $(N P)$. Generally, during the iterative process of the
SQP method, the next iterate point is generated by solving a subproblem as follows:

$$
\begin{aligned}
\max _{y \in S(x)}\{ & \langle\nabla f(x), x-y\rangle \\
& \left.-\frac{1}{2}\langle B(x)(y-x), y-x\rangle\right\},
\end{aligned}
$$

where

$$
\begin{gathered}
S(x)=\left\{y \in \mathbf{R}^{n} \mid\left\langle\nabla c_{i}(x), y-x\right\rangle \leq 0, i \in I_{S}(x)\right\}, \\
I_{S}(x)=\left\{1 \leq i \leq m \mid c_{i}(x)=0\right\},
\end{gathered}
$$

$\nabla f$ and $\nabla c_{i}$ are gradients of $f$ and $c_{i}$, respectively, and $B(x)$ is a symmetric positive definite matrix. The matrix $B(x)$ is modified and selected again along with the iterative process.

For the matrix $B(x)$, we assume in this paper that there exist positive numbers $\lambda_{\min }$ and $\lambda_{\max }$ such that

$$
\lambda_{\min } \leq\|B(x)\| \leq \lambda_{\max }, \quad \forall x \in S,
$$

where $\|\cdot\|$ stands for the Euclidean norm.

Due to the convexity of $c_{i}(\cdot)$ and $I_{S}(x) \subseteq\{1,2, \ldots, m\}$, it is easy to see that $S \subseteq S(x)$ for all $x \in S$. 
Consider the following function:

$$
\begin{aligned}
\phi(x)=\max _{y \in S(x)}\{ & \langle\nabla f(x), x-y\rangle \\
& \left.-\frac{1}{2}\langle B(x)(y-x), y-x\rangle\right\},
\end{aligned}
$$

which is referred to as a merit function.

It is well known that the SQP method has wide and valid application for solving optimization problems (see [1$7]$ ). However, the aim of this paper is not to propose more algorithms or computing skills for SQP methods but to study the global error bounds of projected gradient for nonconvex problems (NP) and the local error bounds of the distance from a feasible solution to the optimal solution set for convex problems $(N P)$, with these error bounds by means of the merit function $\phi(x)$ being provided. This is one of the main contents of this paper.

The theory of error bounds has attracted a lot of attention and many good results have been obtained. In particular, [8, 9] established many types of global error bounds for monotone affine variational inequality problems; [10-12] developed global $S$-type error bounds and global error bounds in $\mathbf{R}^{n}$ for strongly monotone variational inequality problems; [13, 14] obtained global $S$-type error bounds for generalized linear complementarity problems and monotone variational inequality problems. However, to the best of our knowledge, there is not much work concerning the error bounds in SQP methods. This motivates us to do this research.

It should be noted that the merit function $\phi(x)$ considered here is different from the regular gap function (another kind of merit function) discussed in $[10,11,15,16]$, since $\phi(x)$ is generated over a polyhedral set obtaining $S$, rather than over $S$ itself. The main advantage of this modification is that the $K K T$ point of the problem $(N P)$ is equivalent to $\phi(x)=0$. In addition, the computation of $\phi(x)$ is easier than the regular gap function.

Another main contribution of this paper is the finite termination of a feasible solution sequence; that is, the feasible solution sequence converges finitely to the solution sets (stationary points set and $K K T$ point set) of problem $(N P)$. This research has received considerable attention (see [17-25]). Among these, under the assumption that the solution set is weak sharp minima or nondegeneration, [20-25] studied the finite termination, respectively; [17-19] discussed, under the stronger condition, finite termination for some new efficient algorithms. It is worth mentioning that Burke and Ferris (see [20]), under the solution set satisfying the condition of weak sharp minima and other hypotheses for convex optimization problems, put forward the necessary and sufficient condition by which a feasible solution sequence converges finitely to the solution set (see [20, Theorem 4.7]). Afterwards, under the same conditions, [21] verified the conclusion in Pseudomonotone ${ }^{+}$variational inequality problems. Recently, [25, Theorem 2] simplified the conditions of [20, Theorem 4.7] and confirmed that [20, Theorem 4.7] is efficacious only under the solution set satisfying the condition of weak sharp minima. However, for nonconvex optimization problems $(N P)$, when its solution set satisfies the condition of weak sharp minima or nondegeneration, establishing the necessary and sufficient condition of a feasible solution sequence to converge finitely to the solution set is undoubtedly of great significance to solve this problem, but up to now we have not seen the research literature on this issue.

In this paper, inspired by [25, Theorem 2], we solve this problem. We firstly extend the concepts of nondegeneration and weak sharp minima and, for the solution set of the problem (NP), establish the definitions of generalized nondegeneration and generalized weak sharp minima. Based on these two generalized concepts, we prove the following main results:

(1) the necessary and sufficient condition of a feasible solution sequence to converge finitely to the solution set for the problem $(N P)$ is that the corresponding sequence of projected gradient converges to zero.

When the feasible solution set $S$ of $(N P)$ is general closed convex, the calculation of gradient projection is very difficult. However, the calculation of a merit function is much easier, and $\phi(x)=0$ equivalent to $x$ being a KKT point. Based on this feature of $\phi(x)$, we characterize the necessary and sufficient condition for the sequence terminating finitely to generalized a nondegeneration $K K T$ point set by global error bound of projected gradient using merit function $\phi(x)$; that is,

(2) for the problem $(N P)$, the necessary and sufficient condition for the feasible solution sequence terminating finitely to generalized nondegeneration $K K T$ point set is that the corresponding sequence of merit function converges to zero.

For generalized weak sharp minima, we

(3) suppose the stationary point set of the nonconvex optimization problem $(N P)$ is convex; then the necessary and sufficient condition for the feasible solution sequence terminating finitely to a generalized weak sharp minima stationary point set is that the corresponding sequence of projected gradient converges to zero. As a straightforward corollary of the result, we obtain [16, Theorem 2]; therefore, we extend [20, Theorem 4.7] to nonconvex optimization problems.

The rest of this paper is organized as follows. In Section 2, we introduce some concepts and symbols which shall be used in the following discussion. In Section 3, we develop some basic properties of $\phi(x)$ and obtain a global error bound for the projected gradient and a local error bound for the distance from a feasible solution to the optimal solution set of problem $(N P)$ by using $\phi(x)$. Finally, in Section 4 , we give the necessary and sufficient condition for the feasible solution sequence terminating finitely to the sets of generalized nondegenerate and weak sharp minima, respectively, and give a number of meaningful conclusions.

\section{Definitions and Notation}

Let $\|\cdot\|$ and $\langle\cdot, \cdot\rangle$ stand for the standard Euclidean norm and inner product in $\mathbf{R}^{n}$, respectively. Denote by $\bar{S}, \widehat{S}$, and $S^{*}$ the stationary points set, $K K T$ points set, and (global) optimal solutions set of problem $(N P)$, respectively. In view 
of the assumption given in the matrix $B(x)$, we know that the optimal solution of the subproblem $(S P(x))$ is unique; say $y^{*}(x)$. For simplicity, we denote this optimal solution by $y^{*}$, and the confusion can be eliminated from the context.

Given a nonempty subset $A$ of $\mathbf{R}^{n}$, its closure set is denoted by $c l A$ and the polar cone is defined as

$$
A^{\circ}=\left\{y \in \mathbf{R}^{n} \mid\langle y, x\rangle \leq 0, \forall x \in A\right\} .
$$

The tangent cone of $A$ at $x \in c l A$ is given by

$$
\begin{aligned}
T_{A}(x)= & \left\{d \in \mathbf{R}^{n} \mid \exists \lambda_{k} \downarrow 0, d^{k} \longrightarrow d,\right. \\
& \text { such that } \left.x+\lambda_{k} d^{k} \in A, \forall k\right\} .
\end{aligned}
$$

The normal cone of $A$ at $x \in A$ is defined as $N_{A}(x)=T_{A}(x)^{\circ}$. In particular, if $A$ is convex, then the tangent and normal cone of $A$ at $x \in A$ take the following form, respectively:

$$
\begin{gathered}
T_{A}(x)=c l\{\lambda(y-x) \mid \lambda \geq 0, y \in S\} ; \\
N_{A}(x)=\left\{y \in \mathbf{R}^{n} \mid\langle y, z-x\rangle \leq 0, \forall z \in A\right\} .
\end{gathered}
$$

The projection of a point $x \in \mathbf{R}^{n}$ onto a closed set $A$ is defined by

$$
P(x \mid A)=\arg \min \{\|y-x\| \mid y \in A\},
$$

and the distance from $x \in \mathbf{R}^{n}$ to $A$ is given by

$$
\operatorname{dist}(x, A)=\|x-P(x \mid A)\| \text {. }
$$

Fixed vector $a \in \mathbf{R}^{n}$ and a nonnegative scalar $t$, we use notation $a+t \mathbf{B}$ to mean that $\left\{x \in \mathbf{R}^{n} \mid\|x-a\| \leq t\right\}$, where $\mathbf{B}$ stands for the unit ball in $\mathbf{R}^{n}$.

A mapping $\nabla_{A} f(\cdot): \mathbf{R}^{n} \rightarrow \mathbf{R}^{n}$ is said to be a projected gradient of $f(\cdot)$ with respect to a set $A$ if

$$
\nabla_{A} f(x)=P\left(-\nabla f(x) \mid T_{A}(x)\right), \quad \forall x \in A .
$$

Clearly, $x \in S$ is a stationary point of $(N P)$ if and only if $-\nabla f(x) \in N_{S}(x)$ or, equivalently, $\nabla_{S} f(x)=0$. Since $c_{i}(x)$ is convex, it is easy to see that $\widehat{S} \subseteq \bar{S}$ and $S^{*} \subseteq \bar{S}$. Clearly, we have $S^{*}=\bar{S}$ for convex optimization problem.

For the solution set $S^{*}$ of optimization problem $(N P)$, Burke and Ferris [20] extended the concept of strong singleton minima point to weak sharp minima, which can be used to deal with the case where the solution set is a nonsingleton point set. More precisely, a set $S^{*}$ is said to be weak sharp minima if there exists a positive $\alpha^{*}$ which depends only on $f, S^{*}$, and $S$, such that

$$
f(x)-f\left(x^{*}\right) \geq \alpha^{*} \operatorname{dist}\left(x, S^{*}\right), \quad \forall x^{*} \in S^{*}, x \in S .
$$

We call that a sequence $\left\{x_{k}\right\}$ terminates finitely to $A$ if there exists $k_{0}$ such that $x_{k} \in A$ for all $k \geq k_{0}$. Given $\alpha \in \mathbf{R}$, the level set of $\phi(x)$ is defined as

$$
L_{\phi}(\alpha)=\{x \in S \mid \phi(x) \leq \alpha\}
$$

\section{Error Bound}

3.1. Properties of $\phi(x)$. This subsection mainly deals with the basic properties of the merit function $\phi(x)$. From the definition of subproblem $(S P(x))$, we know that $\phi(x) \geq 0$ for all $x \in S$. Since $S(x)$ is polyhedral, we have the following.

Lemma 1. Given $x \in S$, a point $y^{*} \in S(x)$ is the unique solution of the subproblem $(S P(x))$ if and only if there exist KKT multipliers $\lambda_{i}^{*} \geq 0$ for $i \in I_{S}(x)$ such that

$$
\begin{gathered}
\nabla f(x)+B(x)\left(y^{*}-x\right)+\sum_{i \in I_{S}(x)} \lambda_{i}^{*} \nabla c_{i}(x)=0, \\
\lambda_{i}^{*}\left\langle\nabla c_{i}(x), y^{*}-x\right\rangle=0, \quad \forall i \in I_{S}(x),
\end{gathered}
$$

where, for simplicity, one uses $\lambda_{i}^{*}$ to denote KKT multipliers $\lambda_{i}^{*}(x)$ associated with the unique optimal solution to the problem $(S P(x))$.

Lemma 2. For any $x \in S$, one has

$$
\phi(x)=\frac{1}{2}\left\langle B(x)\left(y^{*}-x\right), y^{*}-x\right\rangle .
$$

Proof. Left-multiplying the two sides of (13) by $\left(x-y^{*}\right)^{T}$ and using (14), we have

$$
\left\langle\nabla f(x), x-y^{*}\right\rangle-\left\langle B(x)\left(y^{*}-x\right), y^{*}-x\right\rangle=0 .
$$

By the definition of $\phi(x)$, we obtain

$$
\begin{aligned}
\phi(x) & =\left\langle\nabla f(x), x-y^{*}\right\rangle-\frac{1}{2}\left\langle B(x)\left(y^{*}-x\right), y^{*}-x\right\rangle \\
& =\frac{1}{2}\left\langle B(x)\left(y^{*}-x\right), y^{*}-x\right\rangle .
\end{aligned}
$$

Lemma 3. For any $x \in S$, one has

$$
\phi(x) \geq \frac{1}{2} \lambda_{\min }\left\|y^{*}-x\right\|^{2} .
$$

With the preparation of these lemmas, we obtain the following result.

Theorem 4. The following conclusions are equivalent:

(1) $x$ is a KKT point of the problem (NP);

(2) $x \in S$ and $\phi(x)=0$;

(3) $x \in S$ and $x=y^{*}(x)$.

From conclusions (1) and (2) of Theorem 4 and the nonnegativity of $\phi(x)$, it is immediate to verify the following result.

Corollary 5. Suppose that the KKT point of (NP) is nonempty. Then, $x^{*}$ is a KKT point of problem (NP) if and only if $x^{*}$ is an optimal solution of problem

$$
\min \{\phi(x) \mid x \in S\} .
$$

In this case, $\phi\left(x^{*}\right)=0$ and $x^{*}=y^{*}\left(x^{*}\right)$. 
Lemma 6. For any $x \in S$ and $z \in S(x)$, one has

$$
\left\langle\nabla f(x)+B(x)\left(y^{*}-x\right), z-y^{*}\right\rangle \geq 0 .
$$

Proof. Clearly, the optimal solutions of problem $(S P(x))$ is the same to that of the following programming problem:

$$
\min _{y \in S(x)}\left\{\langle\nabla f(x), y-x\rangle+\frac{1}{2}\langle B(x)(y-x), y-x\rangle\right\} .
$$

Since this is a convex programming, its optimal solutions must be stationary points as well; that is,

$$
\left\langle\nabla f(x)+B(x)\left(y^{*}-x\right), z-y^{*}\right\rangle \geq 0 .
$$

3.2. Error Bound. The following result provides an estimation for $\left\|\nabla_{S(x)} f(x)\right\|$.

Theorem 7. For any $x \in S$, one has

$$
\beta_{2} \phi(x)^{1 / 2} \leq\left\|\nabla_{S(x)} f(x)\right\| \leq \beta_{1} \phi(x)^{1 / 2},
$$

where $\beta_{1}=\lambda_{\max }\left(2 / \lambda_{\min }\right)^{1 / 2}$ and $\beta_{2}=\left(\lambda_{\min } / 2\right)^{1 / 2}$.

Proof. Since $S(x)$ is a polyhedral set, it is easy to see that the tangent cone of $S(x)$ at $x$ takes the form as follows:

$$
\begin{aligned}
T_{S(x)}(x) & =\left\{d \in \mathbf{R}^{n} \mid\left\langle\nabla c_{i}(x), d\right\rangle \leq 0, i \in I_{S(x)}(x)\right\} \\
& =\left\{d \in \mathbf{R}^{n} \mid\left\langle\nabla c_{i}(x), d\right\rangle \leq 0, i \in I_{S}(x)\right\},
\end{aligned}
$$

where we have used the fact that $I_{S}(x)=I_{S(x)}(x)$. Take $d \epsilon$ $T_{S(x)}(x)$ with $\|d\| \leq 1$. Left-multiplying both sides of (13) by $d^{T}$ and using Lemma 3, we obtain

$$
\begin{aligned}
\langle-\nabla f(x), d\rangle & =\left\langle B(x)\left(y^{*}-x\right), d\right\rangle+\sum_{i \in I_{S}(x)} \lambda_{i}^{*}\left\langle\nabla c_{i}(x), d\right\rangle \\
& \leq\left\langle B(x)\left(y^{*}-x\right), d\right\rangle \\
& \leq \lambda_{\max }\left\|y^{*}-x\right\| \\
& \leq \lambda_{\max }\left(\frac{2}{\lambda_{\min }}\right)^{1 / 2} \phi(x)^{1 / 2} .
\end{aligned}
$$

In other words, we obtain

$$
\begin{array}{r}
\langle-\nabla f(x), d\rangle \leq \beta_{1} \phi(x)^{1 / 2}, \\
\forall d \in T_{S(x)}(x) \text { with }\|d\| \leq 1,
\end{array}
$$

where $\beta_{1}=\lambda_{\max }\left(2 / \lambda_{\min }\right)^{1 / 2}$. This, together with the properties of projected gradient by Calamai and Moré [26], implies that

$$
\begin{aligned}
\left\|\nabla_{S(x)} f(x)\right\| & =\max \left\{\langle-\nabla f(x), d\rangle \mid d \in T_{S(x)}(x),\|d\| \leq 1\right\} \\
& \leq \beta_{1} \phi(x)^{1 / 2} .
\end{aligned}
$$

Thus, the right inequality is proved.
On the other hand, Lemma 3 implies that

$$
\left(\frac{\lambda_{\min }}{2}\right)^{1 / 2}\left\|y^{*}-x\right\| \leq \phi(x)^{1 / 2}
$$

from which we obtain

$$
\left(\frac{\lambda_{\min }}{2}\right)^{1 / 2} \phi(x)^{1 / 2} \leq \frac{1}{\left\|y^{*}-x\right\|} \phi(x) .
$$

Let $\beta_{2}=\left(\lambda_{\min } / 2\right)^{1 / 2}$. Note that

$$
\begin{gathered}
\frac{y^{*}-x}{\left\|y^{*}-x\right\|} \in T_{S(x)}(x), \\
\left\|\frac{y^{*}-x}{\left\|y^{*}-x\right\|}\right\|=1 .
\end{gathered}
$$

From (29) and (30), we obtain

$$
\begin{aligned}
\beta_{2} \phi(x)^{1 / 2} \leq & \frac{\phi(x)}{\left\|y^{*}-x\right\|} \\
= & \left\langle-\nabla f(x), \frac{y^{*}-x}{\left\|y^{*}-x\right\|}\right\rangle \\
& -\frac{1}{2}\left\langle B(x)\left(y^{*}-x\right), \frac{y^{*}-x}{\left\|y^{*}-x\right\|}\right\rangle \\
\leq & \left\langle-\nabla f(x), \frac{y^{*}-x}{\left\|y^{*}-x\right\|}\right\rangle \\
\leq & \max \left\{\langle-\nabla f(x), d\rangle \mid d \in T_{S(x)}(x),\|d\| \leq 1\right\} \\
= & \left\|\nabla_{S(x)} f(x)\right\| .
\end{aligned}
$$

So, the left inequality is valid.

The following result can be obtained by Theorems 4 and 7.

Corollary 8. The following conclusions are equivalent:

(1) $x$ is a KKT point of the problem (NP);

(2) $x \in S$ and $\nabla_{S(x)} f(x)=0$.

A global error bound for $\nabla_{S} f(x)$ is given below.

Theorem 9. For any $x \in S$, one has

$$
\left\|\nabla_{S} f(x)\right\| \leq \beta_{1} \phi(x)^{1 / 2} .
$$

If $S$ is polyhedral, then

$$
\beta_{2} \phi(x)^{1 / 2} \leq\left\|\nabla_{S} f(x)\right\| \leq \beta_{1} \phi(x)^{1 / 2},
$$

where $\beta_{1}=\lambda_{\max }\left(2 / \lambda_{\min }\right)^{1 / 2}$ and $\beta_{2}=\left(\lambda_{\min } / 2\right)^{1 / 2}$. 
Proof. Since $S \subseteq S(x)$ and $x \in S$, it follows from the definition of tangent cone that

$$
T_{S}(x) \subseteq T_{S(x)}(x)
$$

Taking into account of the properties of the projected gradient and Theorem 7, we obtain

$$
\begin{aligned}
\left\|\nabla_{S} f(x)\right\| & =\max \left\{\langle-\nabla f(x), d\rangle \mid d \in T_{S}(x),\|d\| \leq 1\right\} \\
& \leq \max \left\{\langle-\nabla f(x), d\rangle \mid d \in T_{S(x)}(x),\|d\| \leq 1\right\} \\
& =\left\|\nabla_{S(x)} f(x)\right\| \\
& \leq \beta_{1} \phi(x)^{1 / 2} .
\end{aligned}
$$

If $S$ is polyhedral, then $T_{S}(x)=T_{S(x)}(x)$; that is, $\nabla_{S} f(x)=\nabla_{S(x)} f(x)$. Thus, the result is established by invoking Theorem 7 .

Now, we consider the case where the problem $(N P)$ is convex; that is, the functions involved in $(N P)$ are all convex. Obviously, in this case, $S^{*}$ is a closed convex set. Under condition (11), we use $\phi(x)$ to give a local error bound for $\operatorname{dist}\left(x, S^{*}\right)$

Theorem 10. Suppose that $(N P)$ is a convex programming. If $S^{*}$ is a set of weak sharp minima; that is, (11) holds, then there exist positive constants $\alpha, \beta$, and $\gamma$ such that

$$
\beta \operatorname{dist}\left(x, S^{*}\right) \leq \gamma \phi(x), \quad \forall x \in L_{\phi}(\alpha) .
$$

Proof. Given any $x \in S$, let $z=P\left(x \mid S^{*}\right)$. Taking into account Lemma 6, we have

$$
\begin{aligned}
& \left\langle\nabla f(x), P\left(x \mid S^{*}\right)-y^{*}\right\rangle \\
& \quad \geq\left\langle B(x)\left(y^{*}-x\right), y^{*}-P\left(x \mid S^{*}\right)\right\rangle .
\end{aligned}
$$

By (11), (37), the convexity of $f(\cdot)$, and the assumption of $B(x)$, we have

$$
\begin{aligned}
\phi(x)= & \left\langle\nabla f(x), x-y^{*}\right\rangle-\frac{1}{2}\left\langle B(x)\left(y^{*}-x\right), y^{*}-x\right\rangle \\
\geq & \left\langle\nabla f(x), x-y^{*}\right\rangle-\frac{1}{2} \lambda_{\max }\left\|y^{*}-x\right\|^{2} \\
= & \left\langle\nabla f(x), x-P\left(x \mid S^{*}\right)\right\rangle+\left\langle\nabla f(x), P\left(x \mid S^{*}\right)-y^{*}\right\rangle \\
& -\frac{1}{2} \lambda_{\max }\left\|y^{*}-x\right\|^{2} \\
\geq & f(x)-f\left(P\left(x \mid S^{*}\right)\right) \\
& +\left\langle B(x)\left(y^{*}-x\right), y^{*}-P\left(x \mid S^{*}\right)\right\rangle \\
& -\frac{1}{2} \lambda_{\max }\left\|y^{*}-x\right\|^{2}
\end{aligned}
$$

$$
\begin{aligned}
\geq & \alpha^{*} \operatorname{dist}\left(x, S^{*}\right)-\lambda_{\max }\left\|y^{*}-x\right\| \\
\cdot & \left\|y^{*}-P\left(x \mid S^{*}\right)\right\|-\frac{1}{2} \lambda_{\max }\left\|y^{*}-x\right\|^{2} \\
\geq & \alpha^{*} \operatorname{dist}\left(x, S^{*}\right)-\lambda_{\max }\left\|y^{*}-x\right\| \operatorname{dist}\left(x, S^{*}\right) \\
& -\frac{3}{2} \lambda_{\max }\left\|y^{*}-x\right\|^{2} .
\end{aligned}
$$

According to the last inequality above and Lemma 3,

$$
\begin{aligned}
\phi(x) \geq & \alpha^{*} \operatorname{dist}\left(x, S^{*}\right)-\lambda_{\max }\left\|y^{*}-x\right\| \operatorname{dist}\left(x, S^{*}\right) \\
& -\frac{3}{2} \lambda_{\max }\left\|y^{*}-x\right\|^{2} \\
\geq & \alpha^{*} \operatorname{dist}\left(x, S^{*}\right)-\lambda_{\max }\left(\frac{2}{\lambda_{\min }}\right)^{1 / 2} \phi(x)^{1 / 2} \operatorname{dist}\left(x, S^{*}\right) \\
& -\frac{3 \lambda_{\max }}{\lambda_{\min }} \phi(x),
\end{aligned}
$$

which in turn implies that

$$
\begin{aligned}
{\left[\alpha^{*}\right.} & \left.-\lambda_{\max }\left(\frac{2}{\lambda_{\min }}\right)^{1 / 2} \phi(x)^{1 / 2}\right] \operatorname{dist}\left(x, S^{*}\right) \\
& \leq\left(\frac{3 \lambda_{\max }}{\lambda_{\min }}+1\right) \phi(x) .
\end{aligned}
$$

Let $\alpha^{1 / 2}=\left(\alpha^{*} / 2\right)\left[\lambda_{\max }\left(2 / \lambda_{\min }\right)^{1 / 2}\right]^{-1}, \beta=(1 / 2) \alpha^{*}$ and $\gamma=$ $3 \lambda_{\text {max }} / \lambda_{\text {min }}+1$. When $x \in L_{\phi}(\alpha)$, it follows from (40) that

$$
\begin{aligned}
\beta \operatorname{dist}\left(x, S^{*}\right) & =\left[\alpha^{*}-\left(\lambda_{\max }\left(\frac{2}{\lambda_{\min }}\right)^{1 / 2}\right) \alpha^{1 / 2}\right] \operatorname{dist}\left(x, S^{*}\right) \\
& \leq\left[\alpha^{*}-\lambda_{\max }\left(\frac{2}{\lambda_{\min }}\right)^{1 / 2} \phi(x)^{1 / 2}\right] \operatorname{dist}\left(x, S^{*}\right) \\
& \leq \gamma \phi(x) .
\end{aligned}
$$

Corollary 11. Suppose that $(N P)$ is a convex programming. If (11) holds and $\left\{x_{k}\right\}$ satisfies $\lim _{k \rightarrow \infty} \phi\left(x_{k}\right)=0$, then

$$
\lim _{k \rightarrow \infty} \operatorname{dist}\left(x_{k}, S^{*}\right)=0 .
$$

Proof. For the positive $\alpha$ given in Theorem 10, it follows from $\lim _{k \rightarrow \infty} \phi\left(x_{k}\right)=0$ that there exists $k_{0}$ such that $\phi\left(x_{k}\right) \leq \alpha$ for all $k \geq k_{0}$; that is, $x_{k} \in L_{\phi}(\alpha)$. The result then follows readily from Theorem 10 .

\section{Finite Termination}

In this section, we will study the necessary and sufficient conditions for the feasible solution sequence of nonconvex optimization problems (NP) terminating finitely to their solution sets (stationary points set and $K K T$ points set). 
4.1. Generalized Nondegenerate Set. First we introduce the concept of nondegenerate set.

Definition 12. Let $S^{\circ} \subset S$; if

$$
-\nabla f(x) \in \operatorname{int} N_{S}(x), \quad \forall x \in S^{\circ}
$$

$S^{\circ}$ is said to be a nondegenerate set.

Now, we further extend the definition of the nondegeneration. Let $N \subseteq\{1,2, \ldots\}$ be an infinite subset.

Definition 13. Let $S^{\circ} \subset S$ and $\left\{x_{k}\right\} \subset \mathbf{R}^{n} . S^{\circ}$ is said to be a generalized nondegenerate set for $\left\{x_{k}\right\}$, if, for every subsequence $\left\{x_{k}\right\}_{k \in N}$, there exists $x_{N} \in S^{\circ}$ and $P_{N} \in \mathbf{R}^{n}$ such that

(i) $-P_{N} \in \operatorname{int} N_{S}\left(x_{N}\right)$;

(ii) $\lim \sup _{k \in N, k \rightarrow \infty}\left(1 /\left\|x_{k}-x_{N}\right\|\right)\left\langle\nabla f\left(x_{k}\right)-P_{N}, x_{k}-\right.$ $\left.x_{N}\right\rangle \geq 0$.

It is easy to verify that the following several propositions expressed are special cases of generalized nondegenerate set.

Proposition 14. Let $S^{\circ} \subset S$ be a nondegenerate set, and let $\left\{x_{k}\right\} \subset \mathbf{R}^{n}$ be a bounded sequence, with its every accumulation point $x^{*} \in S^{\circ}$. Then, $S^{\circ}$ is a generalized nondegenerate set for $\left\{x_{k}\right\}$.

Proposition 15. Let $S^{\circ} \subset S,\left\{x_{k}\right\} \subset \mathbf{R}^{n}$, and $\left\{\nabla f\left(x_{k}\right)\right\}$ be bounded. If, for every accumulation point $\nabla f^{*}$ of $\left\{\nabla f\left(x_{k}\right)\right\}$, there exists $x^{*} \in S^{\circ}$ such that

$$
-\nabla f^{*} \in \operatorname{int} N_{S}\left(x^{*}\right)
$$

then $S^{\circ}$ is a generalized nondegenerate set for $\left\{x_{k}\right\}$.

From [20, Theorem 3.1], we know that gradient $\nabla f(\cdot)$ is always constant vector in the optimal solution set of a convex optimization problems. So, according to the monotonicity of $\nabla f(\cdot)$, we get the following.

Proposition 16. Let $S^{*}$, the optimal solution set of a convex optimization problems (NP), be a nondegenerate set. Then, for any $\left\{x_{k}\right\} \subset \mathbf{R}^{n}, S^{*}$ is a generalized nondegenerate set for $\left\{x_{k}\right\}$.

Here, we give the necessary and sufficient condition for the feasible solution sequence of the nonconvex optimization problems $(N P)$ terminating finitely to its generalized nondegenerate solution set.

Theorem 17. For the nonconvex optimization problem (NP), let $\left\{x_{k}\right\} \subset S$, the solution set $(\bar{S}$ or $\widehat{S})$, be a generalized nondegenerate set. Then, $\left\{x_{k}\right\}$ terminates finitely to solution set, if and only if

$$
\lim _{k \rightarrow \infty} \nabla_{S} f\left(x_{k}\right)=0
$$

Proof. Consider the following.

Necessity. If $x_{k} \in \bar{S}$, by the definition of stationary point, we have $\nabla_{S} f\left(x_{k}\right)=0$. If $x_{k} \subseteq \widehat{S}$, for $\widehat{S} \subset \bar{S}$, we also have $\nabla_{S} f\left(x_{k}\right)=$ 0 .

Sufficiency. We only give the proof for $\bar{S}$. The proof for $\widehat{S}$ is the same as that for $\bar{S}$. Suppose (45) holds. We prove the result by contradiction. Suppose on the contrary that there exists an infinite subsequence $N \subseteq\{1,2, \ldots\}$ such that

$$
x_{k} \notin \bar{S}, \quad \forall k \in N \text {. }
$$

Since $\bar{S}$ is a generalized nondegenerate for $\left\{x_{k}\right\}$, so, according to Definition 13, for subsequence $\left\{x_{k}\right\}_{k \in N}, \exists x_{N} \in \bar{S}$ and $P_{N} \in$ $\mathbf{R}^{n}$, such that

$$
-P_{N} \in \operatorname{int} N_{S}\left(x_{N}\right)
$$

$$
\lim _{k \in N, k \rightarrow \infty} \frac{1}{\left\|x_{k}-x_{N}\right\|}\left\langle\nabla f\left(x_{k}\right)-P_{N}, x_{k}-x_{N}\right\rangle \geq 0 .
$$

From (47), we know that $\exists \alpha>0$ such that

$$
-P_{N}+\alpha \mathbf{B} \subset N_{S}\left(x_{N}\right),
$$

where $\mathbf{B}$ stands for the unit ball in $\mathbf{R}^{n}$. By (49), for $\forall g \in$ $T_{S}\left(x_{N}\right), g \neq 0$, we have

$$
\left\langle-P_{N}+\alpha \frac{g}{\|g\|}, g\right\rangle \leq 0
$$

that is,

$$
\alpha \leq\left\langle P_{N}, \frac{g}{\|g\|}\right\rangle .
$$

Let $g_{k}=x_{k}-x_{N}(\forall k \in N)$, and by (46) we have $g_{k} \neq 0$. Since $S$ is convex, we have

$$
g_{k} \in T_{S}\left(x_{N}\right), \quad-g_{k} \in T_{S}\left(x_{k}\right) \quad(\forall k \in N) .
$$

Then, according to (51), (52), and the property of gradient projection, we have for all $k \in N$

$$
\begin{aligned}
\alpha \leq & \left\langle P_{N}, \frac{g_{k}}{\left\|g_{k}\right\|}\right\rangle \\
= & \left\langle-P_{N},-\frac{g_{k}}{\left\|g_{k}\right\|}\right\rangle \\
= & \left\langle-\nabla f\left(x_{k}\right),-\frac{g_{k}}{\left\|g_{k}\right\|}\right\rangle-\left\langle\nabla f\left(x_{k}\right)-P_{N}, \frac{g_{k}}{\left\|g_{k}\right\|}\right\rangle \\
\leq & \max \left\{\left\langle-\nabla f\left(x_{k}\right), d\right\rangle \mid d \in T_{S}\left(x_{k}\right),\|d\| \leq 1\right\} \\
& -\left\langle\nabla f\left(x_{k}\right)-P_{N}, \frac{g_{k}}{\left\|g_{k}\right\|}\right\rangle \\
= & \left\|\nabla_{S} f\left(x_{k}\right)\right\|-\left\langle\nabla f\left(x_{k}\right)-P_{N}, \frac{g_{k}}{\left\|g_{k}\right\|}\right\rangle .
\end{aligned}
$$


It follows that

$$
\alpha \leq\left\|\nabla_{S} f\left(x_{k}\right)\right\|-\left\langle\nabla f\left(x_{k}\right)-P_{N}, \frac{g_{k}}{\left\|g_{k}\right\|}\right\rangle, \quad(\forall k \in N) .
$$

Since $g_{k}=x_{k}-x_{N}$, according to (45) (48), we have

$$
\begin{aligned}
\alpha & \leq \liminf _{k \in N, k \rightarrow \infty}\left\{\left\|\nabla_{S} f\left(x_{k}\right)\right\|-\left\langle\nabla f\left(x_{k}\right)-P_{N}, \frac{g_{k}}{\left\|g_{k}\right\|}\right\rangle\right\} \\
& \leq-\lim _{k \in N, k \rightarrow \infty} \sup _{k \rightarrow \infty}\left\langle\nabla f\left(x_{k}\right)-P_{N}, \frac{g_{k}}{\left\|g_{k}\right\|}\right\rangle \\
& \leq 0,
\end{aligned}
$$

which is a contradiction with $\alpha>0$.

Corollary 18. For the the nonconvex optimization problems $(N P)$, let $\left\{x_{k}\right\} \subset S$ be a bounded sequence and let $\bar{S}$ be a nondegenerate set. Then, $\left\{x_{k}\right\}$ terminates finitely to $\bar{S}$, if and only if (45) holds.

Proof. The Necessity is obvious. We only need to prove the Sufficiency. Suppose (45) holds; that is,

$$
\lim _{k \rightarrow \infty} \nabla_{S} f\left(x_{k}\right)=0
$$

According to the lower semicontinuity of $\nabla_{S} f(\cdot)$ and the boundedness of $\left\{x_{k}\right\}$, we have that every accumulation point $x^{*}$ of $\left\{x_{k}\right\}$ is in $\bar{S}$. Therefore, from Proposition 14, we know that $\bar{S}$ is a generalized nondegenerate set for $\left\{x_{k}\right\}$. Then, according to (45), by Theorem 17, sufficiency holds.

Corollary 19. For the nonconvex optimization problem (NP), let $\left\{x_{k}\right\} \subset S$, and $\left\{\nabla f\left(x_{k}\right)\right\}$ is a bounded sequence, and, for each of its accumulation point $\nabla f^{*}$, there exists $x^{*} \in \bar{S}$ (or $\left.x^{*} \in \widehat{S}\right)$ such that

$$
-\nabla f^{*} \in \operatorname{int} N_{S}\left(x^{*}\right) .
$$

Then, $\left\{x_{k}\right\}$ terminates finitely to the solution set $\bar{S}$ (or $\widehat{S}$ ) if and only if (45) holds.

Proof. By Proposition 15 and Theorem 17, we can get it.

Corollary 20. For the nonconvex optimization problem (NP), let $\left\{x_{k}\right\} \subset S$, and $\bar{S}$ is a nondegenerate set. Then, $\left\{x_{k}\right\}$ terminates finitely to $S^{*}$, if and only if (45) holds.

Proof. Here, we have $S^{*}=\bar{S}$. Then, by Proposition 16 and Theorem 17, we can get it.

In the following, we will use the global error bounds of projected gradient, which resulted from last section, to characterize the necessary and sufficient condition of feasible solution sequence terminating finitely by the merit function $\phi(\cdot)$ which is easy to calculate.
Theorem 21. For the nonconvex optimization problem (NP), $\left\{x_{k}\right\} \subset S$, and KKT points set $\widehat{S}$ is a generalized nondegenerate set for $\left\{x_{k}\right\}$. Then, $\left\{x_{k}\right\}$ terminates finitely to $\widehat{S}$, if and only if

$$
\lim _{k \rightarrow \infty} \phi\left(x_{k}\right)=0
$$

Proof. Consider the following.

Necessity. If $x_{k} \in \widehat{S}$, then, according to Theorem 4, we have $\phi\left(x_{k}\right)=0$.

Sufficiency. Suppose (58) holds, and according to Theorem 9, we have $\lim _{k \rightarrow \infty} \nabla_{S} f\left(x_{k}\right)=0$; that is, (45) holds. From Theorem 17, we get the sufficiency.

Lemma 22. For the nonconvex optimization problem (NP), let $\left\{x_{k}\right\} \subset S$, and (58) holds. If $x^{*}$ is an accumulation point of $\left\{x_{k}\right\}$ satisfying the Mangasarian-Fromovitz constraint qualification, then $x^{*}$ is a KKT point of $(N P)$.

Proof. Without loss of generality, we assume that

$$
\lim _{k \rightarrow \infty} x_{k}=x^{*}
$$

Then, there exists an index set $I_{S} \subset\{1,2, \ldots, m\}$ and an infinite subsequence $N \subseteq\{1,2, \ldots\}$ such that

$$
I_{S}\left(x_{k}\right)=I_{S} \subseteq I_{S}\left(x^{*}\right), \quad \forall k \in N .
$$

The tangent cone of $S(x)$ at $x \in S$ is

$$
T_{S(x)}(x)=\left\{d \in \mathbf{R}^{n} \mid\left\langle\nabla c_{i}(x), d\right\rangle \leq 0, i \in I_{S}(x)\right\},
$$

and its normal cone is

$$
N_{S(x)}(x)=\left\{\sum_{i \in I_{S}(x)} \alpha_{i} \nabla c_{i}(x) \mid \alpha_{i} \geq 0, i \in I_{S}(x)\right\} .
$$

Therefore, by (60) and (62), when $k \in N$, there exists $\lambda_{i}^{(k)} \geq 0$ for $i \in I_{S}$ such that

$$
P\left(-\nabla f\left(x_{k}\right) \mid N_{S\left(x_{k}\right)}\left(x_{k}\right)\right)=\sum_{i \in I_{S}} \lambda_{i}^{(k)} \nabla c_{i}\left(x_{k}\right)
$$

from which, and using the orthogonal projection decomposition, for all $k \in N$, we get

$$
\begin{aligned}
-\nabla f\left(x_{k}\right)= & P\left(-\nabla f\left(x_{k}\right) \mid T_{S\left(x_{k}\right)}\left(x_{k}\right)\right) \\
& +P\left(-\nabla f\left(x_{k}\right) \mid N_{S\left(x_{k}\right)}\left(x_{k}\right)\right) \\
= & \nabla_{S\left(x_{k}\right)} f\left(x_{k}\right)+\sum_{i \in I_{S}} \lambda_{i}^{(k)} \nabla c_{i}\left(x_{k}\right) .
\end{aligned}
$$

We now show that the sequence $\left\{\sum_{i \in I_{S}} \lambda_{i}^{(k)}\right\}_{k \in N}$ must be bounded. Suppose on the contrary that

$$
\lim _{k \in N, k \rightarrow \infty} \sum_{i \in I_{S}} \lambda_{i}^{(k)}=+\infty
$$


Let us suppose

$$
\lim _{k \in N, k \rightarrow \infty} \frac{\lambda_{i}^{(k)}}{\sum_{i \in I_{S}} \lambda_{i}^{(k)}}=\alpha_{i}^{*}, \quad i \in I_{S} .
$$

Dividing the two sides of (64) by $\sum_{i \in I_{S}} \lambda_{i}^{(k)}$ and taking limit, due to (65) and (66), with the continuity of $\nabla c_{i}(\cdot)$, we obtain

$$
\sum_{i \in I_{S}} \alpha_{i}^{*} \nabla c_{i}\left(x^{*}\right)=0,
$$

and, according to (66), we have

$$
\sum_{i \in I_{S}} \alpha_{i}^{*}=1, \quad \alpha_{i}^{*} \geq 0, \quad i \in I_{S} .
$$

According to the assumption, $x^{*}$ satisfies the $M-F$ constraint qualification; that is, there is $h \in \mathbf{R}^{n}$ such that

$$
\left\langle\nabla c_{i}\left(x^{*}\right), h\right\rangle<0, \quad \forall i \in I_{S}\left(x^{*}\right) .
$$

Since $I_{S} \subseteq I_{S}\left(x^{*}\right)$, so (69), (67), and (68) are contradictory. So we assume that $\lim _{k \in N, k \rightarrow \infty} \lambda_{i}^{(k)}=\lambda_{i}^{*}$ for $i \in I_{S}$. By (58) and Theorem 7, we know that $\lim _{k \rightarrow \infty} \nabla_{S\left(x_{k}\right)} f\left(x_{k}\right)=0$. Then, by (64) and the continuity of $\nabla f(\cdot)$, we obtain

$$
\begin{aligned}
-\nabla f\left(x^{*}\right) & =\lim _{k \in N, k \rightarrow \infty}-\nabla f\left(x_{k}\right) \\
& =\lim _{k \in N, k \rightarrow \infty} \nabla_{S\left(x_{k}\right)} f\left(x_{k}\right)+\lim _{k \in N, k \rightarrow \infty} \sum_{i \in I_{S}} \lambda_{i}^{(k)} \nabla c_{i}\left(x_{k}\right) \\
& =\sum_{i \in I_{S}} \lambda_{i}^{*} \nabla c_{i}\left(x^{*}\right),
\end{aligned}
$$

which means that $x^{*}$ is a KKT point of the $(N P)$.

Corollary 23. For the nonconvex optimization problem (NP), suppose $\widehat{S}$ is a nondegenerate set, $\left\{x_{k}\right\} \subset S$ is bounded, and each of its accumulation point $x^{*}$ is satisfying the Mangasarian-Fromovitz constraint qualification. Then, $\left\{x_{k}\right\}$ terminates finitely to $\widehat{S}$ if and only if the sequence satisfies (58).

Proof. According to Lemma 22 and Proposition 14, by Theorem 21 we can get it.

Corollary 24. For the nonconvex optimization problem (NP), suppose $\left\{x_{k}\right\} \subset S$, and $\left\{\nabla f\left(x_{k}\right)\right\}$ is a bounded sequence, and, for each of its accumulation point $\nabla f^{*}$, there exists $x^{*} \in \widehat{S}$ such that

$$
-\nabla f^{*} \in \operatorname{int} N_{S}\left(x^{*}\right) .
$$

Then, $\left\{x_{k}\right\}$ terminates finitely to $\widehat{S}$ if and only if the sequence satisfies (58).

Proof. According to Proposition 15, by Theorem 21 we can get it.
4.2. Generalized Weak Sharp Minima. In [20], Burke and Ferris gave an equivalent condition for weak sharp minima for a convex optimization problem; that is, the optimal solution set $S^{*}$ of the convex optimization problem is a set of weak sharp minima if and only if for every $\bar{x} \in S^{*}$

$$
-\nabla f(\bar{x}) \in \operatorname{int} \bigcap_{x \in S^{*}}\left[T_{S}(x) \cap N_{S^{*}}(x)\right]^{\circ} .
$$

Generally speaking, for a nonconvex optimization problem, (72) is only a necessary condition for weak sharp minima condition (11); that is, (72) is weaker than (11). However, considering its importance in analyzing the finite termination of algorithms, some previous studies, for example, [21, 22], directly use (72) as the definition of weak sharp minima for the optimal solution set. In this paper, we will use the weaker condition (72) to define the set of weak sharp minima.

Definition 25. Let $S^{\circ} \subset S$, and $S^{\circ}$ is said to be a set of weak sharp minima if and only if (72) holds.

Now, we further extend the definition of weak sharp minima as follows.

Definition 26. Let $S^{\circ} \subset S$ and $\left\{x_{k}\right\} \subset \mathbf{R}^{n} . S^{\circ}$ is said to be a set of generalized weak sharp minima for $\left\{x_{k}\right\}$, if, for every subsequence $\left\{x_{k}\right\}_{k \in N}$, there exists $P_{N} \in \mathbf{R}^{n}$ such that

(i) $-P_{N} \in \operatorname{int} \bigcap_{x \in S^{\circ}}\left[T_{S}(x) \cap N_{S^{\circ}}(x)\right]^{\circ}$;

(ii) $\lim \sup _{k \in N, k \rightarrow \infty}\left(1 /\left\|x_{k}-P\left(x_{k} \quad \mid \quad S^{\circ}\right)\right\|\right)\left\langle\nabla f\left(x_{k}\right)-\right.$ $\left.P_{N}, x_{k}-P\left(x_{k} \mid S^{\circ}\right)\right\rangle \geq 0$.

With the same as the set of generalized nondegeneration, it is easy to verify that the following several propositions expressed are special cases of generalized weak sharp minima.

Proposition 27. Let $S^{\circ} \subset S$ be a set of weak sharp minima, and let $\left\{x_{k}\right\} \subset \mathbf{R}^{n}$ be a bounded sequence, with its every accumulation point $x^{*} \in S^{\circ}$. Then, $S^{\circ}$ is a set of generalized weak sharp minima for $\left\{x_{k}\right\}$.

Proposition 28. Let $S^{\circ} \subset S,\left\{x_{k}\right\} \subset \mathbf{R}^{n}$, and $\left\{\nabla f\left(x_{k}\right)\right\}$ be bounded. If, for every accumulation point $\nabla f^{*}$ of $\left\{\nabla f\left(x_{k}\right)\right\}$,

$$
-\nabla f^{*} \in \operatorname{int} \bigcap_{x \in S^{\circ}}\left[T_{S}(x) \cap N_{S^{\circ}}(x)\right]^{\circ}
$$

then, $S^{\circ}$ is a set of generalized weak sharp minima for $\left\{x_{k}\right\}$.

Proposition 29. Let the optimal solution set $S^{*}$ of a convex optimization problem (NP) be a set of weak sharp minima. Then, for any $\left\{x_{k}\right\} \subset \mathbf{R}^{n}, S^{*}$ is a set of generalized weak sharp minima for $\left\{x_{k}\right\}$.

Here, we give the necessary and sufficient condition for the feasible solution sequence of a nonconvex optimization problem $(N P)$ terminating finitely to its solution set of generalized weak sharp minima.

Theorem 30. For the nonconvex optimization problem (NP), let $\left\{x_{k}\right\} \subset S$, and its stationary point set $\bar{S}$ is convex and is 
a set of generalized weak sharp minima for $\left\{x_{k}\right\}$. Then, $\left\{x_{k}\right\}$ terminates finitely to $\bar{S}$, if and only if (45) holds.

Proof. Consider the following.

Necessity. If $x_{k} \in \bar{S}$, by the definition of stationary point, we have $\nabla_{S} f\left(x_{k}\right)=0$.

Sufficiency. Suppose (45) holds. We prove the result by contradiction. Suppose on the contrary that there exists an infinite subsequence $N \subseteq\{1,2, \ldots\}$ such that

$$
x_{k} \notin \bar{S}, \quad \forall k \in N \text {. }
$$

So, according to Definition 26 (i), we know that, for subsequence $\left\{x_{k}\right\}_{k \in N}, \exists P_{N} \in \mathbf{R}^{n}$ and $\alpha>0$, such that, for $\forall x \in \bar{S}$, we have

$$
-P_{N}+\alpha \mathbf{B} \subset\left[T_{S}(x) \cap N_{\bar{S}}(x)\right]^{\circ} .
$$

By (74), for $\forall g \in T_{S}(x) \cap N_{\bar{S}}(x)$ and $\forall x \in \bar{S}$, we have

$$
\alpha \leq\left\langle P_{N}, \frac{g}{\|g\|}\right\rangle .
$$

Let $z_{k}=P\left(x_{k} \mid \bar{S}\right)$ and $g_{k}=x_{k}-z_{k}$. By (74), we have $\forall k \in N$, $g_{k} \neq 0$. Since $S$ is convex, we have

$$
g_{k} \in T_{S}\left(z_{k}\right), \quad-g_{k} \in T_{S}\left(x_{k}\right) \text {. }
$$

Since $\bar{S}$ is convex, according to the property of projection and the definition of $N_{\bar{S}}(\cdot)$, we have $g_{k} \in N_{\bar{S}}\left(z_{k}\right)$, so by (77), we have

$$
g_{k} \in T_{S}\left(z_{k}\right) \cap N_{\bar{S}}\left(z_{k}\right) .
$$

Then, according to (76), (77), (78), and the property of gradient projection, we have

$$
\begin{aligned}
\alpha \leq & \left\langle P_{N}, \frac{g_{k}}{\left\|g_{k}\right\|}\right\rangle \\
= & \left\langle-P_{N},-\frac{g_{k}}{\left\|g_{k}\right\|}\right\rangle \\
= & \left\langle-\nabla f\left(x_{k}\right),-\frac{g_{k}}{\left\|g_{k}\right\|}\right\rangle-\left\langle\nabla f\left(x_{k}\right)-P_{N}, \frac{g_{k}}{\left\|g_{k}\right\|}\right\rangle \\
\leq & \max \left\{\left\langle-\nabla f\left(x_{k}\right), d\right\rangle \mid d \in T_{S}\left(x_{k}\right),\|d\| \leq 1\right\} \\
& -\left\langle\nabla f\left(x_{k}\right)-P_{N}, \frac{g_{k}}{\left\|g_{k}\right\|}\right\rangle \\
= & \left\|\nabla_{S} f\left(x_{k}\right)\right\|-\left\langle\nabla f\left(x_{k}\right)-P_{N}, \frac{g_{k}}{\left\|g_{k}\right\|}\right\rangle .
\end{aligned}
$$

It follows that

$$
\alpha \leq\left\|\nabla_{S} f\left(x_{k}\right)\right\|-\left\langle\nabla f\left(x_{k}\right)-P_{N}, \frac{g_{k}}{\left\|g_{k}\right\|}\right\rangle .
$$

Since $g_{k}=x_{k}-P\left(x_{k} \mid \bar{S}\right)$, according to (45) and Definition 26 (ii), we have

$$
\begin{aligned}
\alpha & \leq \liminf _{k \in N, k \rightarrow \infty}\left\{\left\|\nabla_{S} f\left(x_{k}\right)\right\|-\left\langle\nabla f\left(x_{k}\right)-P_{N}, \frac{g_{k}}{\left\|g_{k}\right\|}\right\rangle\right\} \\
& \leq-\lim _{k \in N, k \rightarrow \infty}\left\langle\nabla f\left(x_{k}\right)-P_{N}, \frac{g_{k}}{\left\|g_{k}\right\|}\right\rangle \\
& \leq 0
\end{aligned}
$$

which is a contradiction with $\alpha>0$.

With the same as generalized nondegeneration, according to Propositions 27, 28, and 29 and by Theorem 30, we can get the following corollaries.

Corollary 31. For the nonconvex optimization problem (NP), suppose $\bar{S}$ is convex and weak sharp minima, and $\left\{x_{k}\right\} \subset S$ is an bounded sequence. Then, $\left\{x_{k}\right\}$ terminates finitely to $\bar{S}$, if and only if (45) holds.

Corollary 32. For the nonconvex optimization problem (NP), suppose $\bar{S}$ is convex and $\left\{x_{k}\right\} \subset S ;\left\{\nabla f\left(x_{k}\right)\right\}$ is a bounded sequence, and for each of its accumulation point $\nabla f^{*}$, we have

$$
-\nabla f^{*} \in \operatorname{int} \bigcap_{x \in \bar{S}}\left[T_{S}(x) \cap N_{\bar{S}}(x)\right]^{\circ} .
$$

Then, $\left\{x_{k}\right\}$ terminates finitely to $\bar{S}$, if and only if (45) holds.

Corollary 33. For the nonconvex optimization problem (NP), the optimal solution set $S^{*}$ is weak sharp minima, $\left\{x_{k}\right\} \subset S$. Then, $\left\{x_{k}\right\}$ terminates finitely to $S^{*}$, if and only if (45) holds.

Notation. Corollary 33 is [25, Theorem 2], which simplifies Theorem 4.7 in Burke and Ferris [20] (which removes two assumptions of [20, Theorem 4.7]; that is, $\lim _{k \rightarrow \infty} \operatorname{dist}\left(x_{k}, S^{*}\right)=0$ and $\nabla f(\cdot)$ is uniformly continuous on an open set containing $\left.\left\{x_{k}\right\}\right)$; therefore, our Theorem 30 extends [20, Theorem 4.7] to nonconvex optimization problems.

\section{Conflict of Interests}

The authors declared that there is no conflict of interests in their submitted paper.

\section{Acknowledgments}

This research was supported by National Natural Science Foundations of China (nos. 11271233 and 10971118) and Natural Science Foundation of Shandong Province (no. ZR2012AM016).

\section{References}

[1] M. Fukushima, Z.-Q. Luo, and J.-S. Pang, "A globally convergent sequential quadratic programming algorithm for mathematical 
programs with linear complementarity constraints," Computational Optimization and Applications, vol. 10, no. 1, pp. 5-34, 1998.

[2] S. J. Wright, "Superlinear convergence of a stabilized SQP method to a degenerate solution," Computational Optimization and Applications, vol. 11, no. 3, pp. 253-275, 1998.

[3] W. W. Hager, "Stabilized sequential quadratic programming," Computational Optimization and Applications, vol. 12, no. 1-3, pp. 253-273, 1999.

[4] T. L. Herdman and K. Kang, "A structured reduced sequential quadratic programming and its application to a shape design problem," Computational Optimization and Applications, vol.11, no. 1, pp. 81-100, 1998.

[5] C. R. Dohrmann and R. D. Robinett, "Efficient sequential quadratic programming implementations for equalityconstrained discrete-time optimal control," Journal of Optimization Theory and Applications, vol. 95, no. 2, pp. 323-346, 1997.

[6] J. V. Burke and S.-P. Han, "A robust sequential quadratic programming method," Mathematical Programming, vol. 43, no. 3, pp. 277-303, 1989.

[7] R. Fletcher, S. Leyffer, D. Ralph, and S. Scholtes, "Local convergence of SQP methods for mathematical programs with equilibrium constraints," SIAM Journal on Optimization, vol. 17, no. 1, pp. 259-286, 2006.

[8] M. S. Gowda and J.-S. Pang, "On the boundedness and stability of solutions to the affine variational inequality problem," SIAM Journal on Control and Optimization, vol. 32, no. 2, pp. 421-441, 1994.

[9] M. C. Ferris and J. S. Pang, "Nondegenerate solutions and related concepts in affine variational inequalities," SIAM Journal on Control and Optimization, vol. 34, no. 1, pp. 244-263, 1996.

[10] J. H. Wu, M. Florian, and P. Marcotte, "A general descent framework for the monotone variational inequality problem," Mathematical Programming, vol. 61, no. 3, pp. 281-300, 1993.

[11] N. Yamashita and M. Fukushima, "Equivalent unconstrained minimization and global error bounds for variational inequality problems," SIAM Journal on Control and Optimization, vol. 35, no. 1, pp. 273-284, 1997.

[12] N. Yamashita, K. Taji, and M. Fukushima, "Unconstrained optimization reformulations of variational inequality problems," Journal of Optimization Theory and Applications, vol. 92, no. 3, pp. 439-456, 1997.

[13] N. H. Xiu and J.Z. Zhang, "Global projection-type error bounds for general variational inequalities," Journal of Optimization Theory and Applications, vol. 112, no. 1, pp. 213-228, 2002.

[14] J. Zhang and N. Xiu, "Global s-type error bound for the extended linear complementarity problem and applications," Mathematical Programming, vol. 88, no. 2, pp. 391-410, 2000.

[15] G. Auchmuty, "Variational principles for variational inequalities," Numerical Functional Analysis and Optimization, vol. 10, no. 9-10, pp. 863-874, 1989.

[16] M. Fukushima, "Equivalent differentiable optimization problems and descent methods for asymmetric variational inequality problems," Mathematical Programming, vol. 53, no. 1, pp. 99110, 1992.

[17] G. H. Lin and M. Fukushima, "Hybrid approach with active set identification for mathematical programs with complementarity constraints," Journal of Optimization Theory and Applications, vol. 128, no. 1, pp. 1-28, 2006.
[18] H.-D. Qi and X.-Q. Yang, "Armijo Newton method for convex best interpolation," Optimization Methods \& Software, vol. 21, no. 2, pp. 179-200, 2006.

[19] M. Kojima and L. Tunçel, "On the finite convergence of successive SDP relaxation methods," European Journal of Operational Research, vol. 143, no. 2, pp. 325-341, 2002.

[20] J. V. Burke and M. C. Ferris, "Weak sharp minima in mathematical programming," SIAM Journal on Control and Optimization, vol. 31, no. 5, pp. 1340-1359, 1993.

[21] P. Marcotte and D. Zhu, "Weak sharp solutions of variational inequalities," SIAM Journal on Optimization, vol. 9, no. 1, pp. 179-189, 1998.

[22] C. Wang, Q. Liu, and X. Yang, "Convergence properties of nonmonotone spectral projected gradient methods," Journal of Computational and Applied Mathematics, vol. 182, no. 1, pp. 5166, 2005.

[23] N. H. Xiu and J. Z. Zhang, "Local convergence analysis of projection-type algorithms: unified approach," Journal of Optimization Theory and Applications, vol. 115, no. 1, pp. 211-230, 2002.

[24] M. V. Solodov and P. Tseng, "Some methods based on the Dgap function for solving monotone variational inequalities," Computational Optimization and Applications, vol. 17, no. 2-3, pp. 255-277, 2000.

[25] J. Zhou and C. Wang, "A note on finite termination of iterative algorithms in mathematical programming," Operations Research Letters, vol. 36, no. 6, pp. 715-717, 2008.

[26] P. H. Calamai and J. J. Moré, "Projected gradient methods for linearly constrained problems," Mathematical Programming, vol. 39, no. 1, pp. 93-116, 1987. 


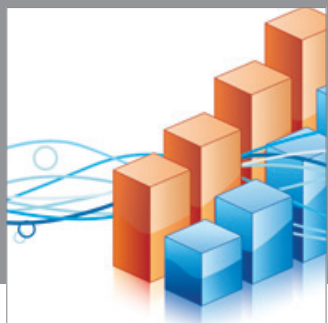

Advances in

Operations Research

mansans

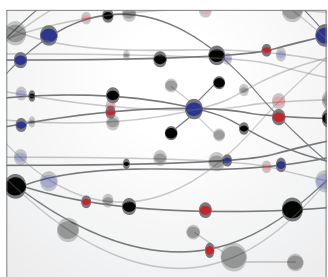

The Scientific World Journal
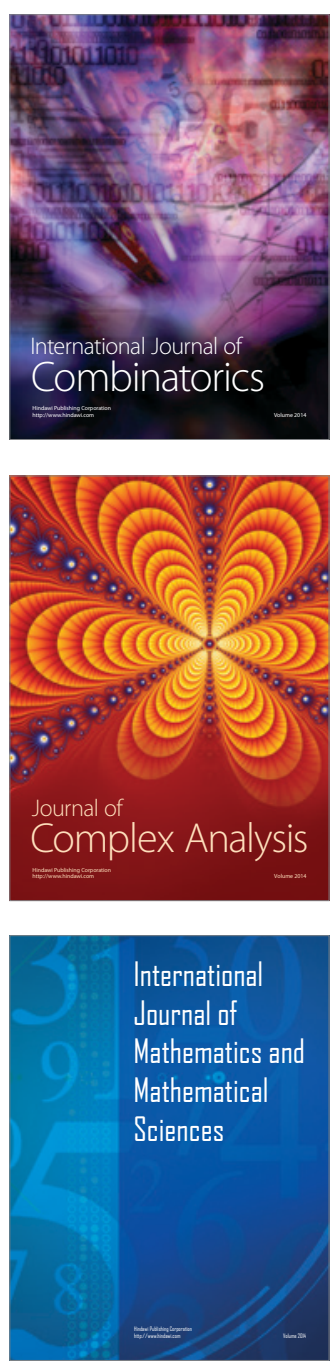
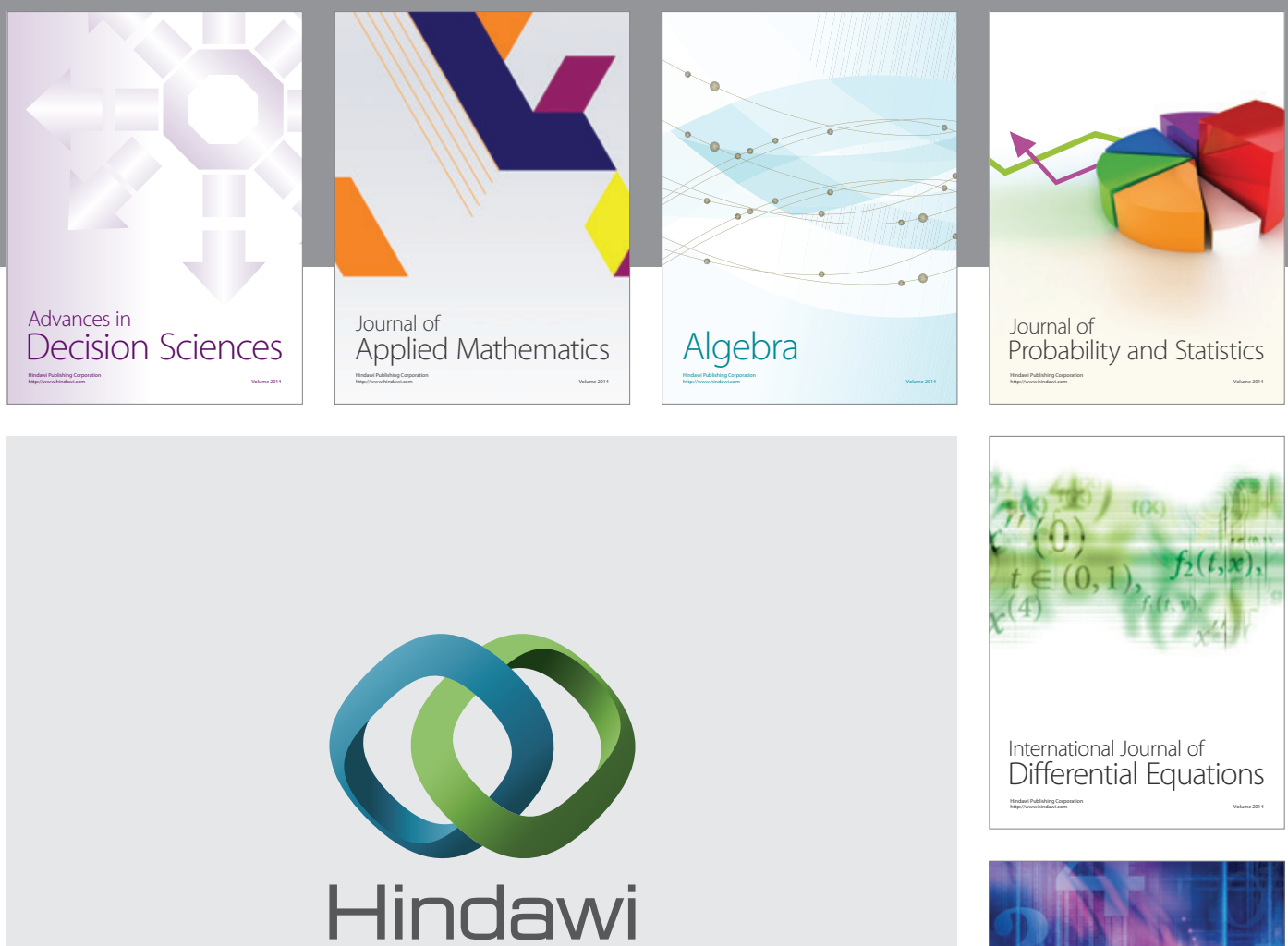

Submit your manuscripts at http://www.hindawi.com
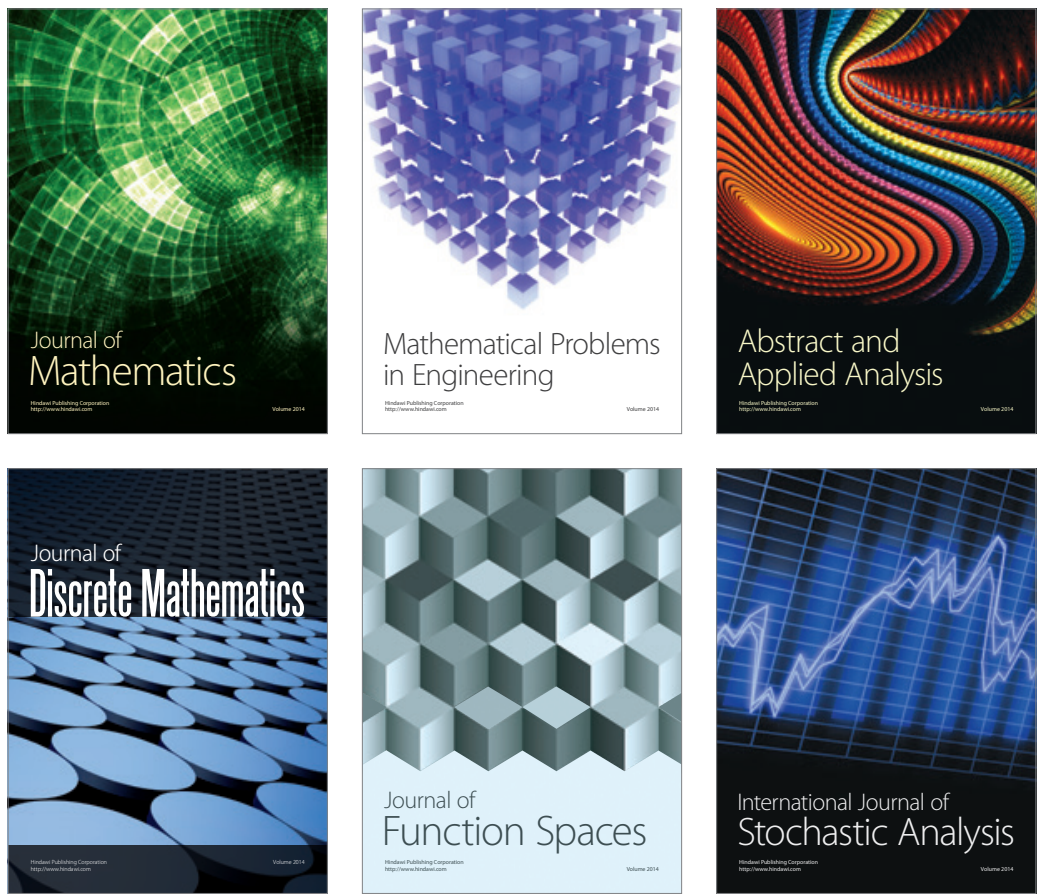

Journal of

Function Spaces

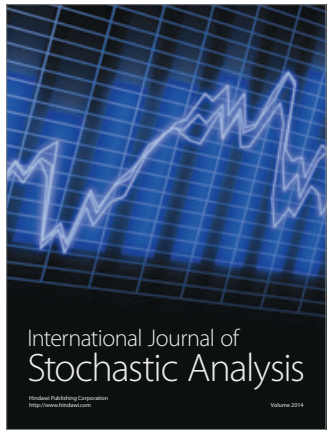

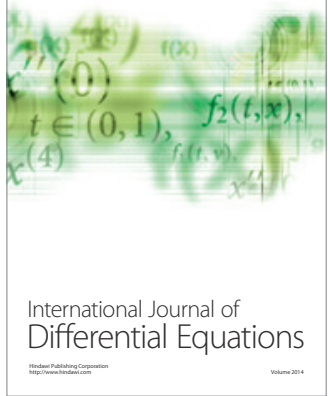
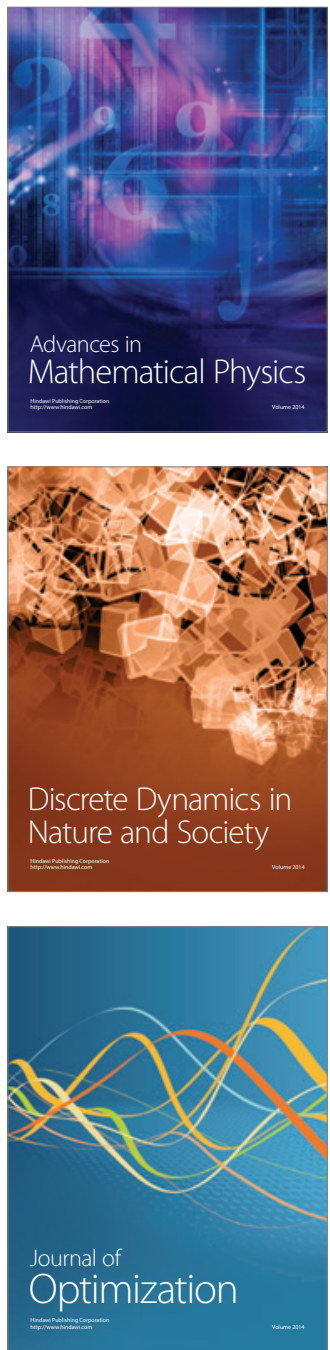